\title{
Murciélagos (Chiroptera: Mammalia) del Parque Nacional Yurubí, Venezuela: listado taxonómico y estudio comunitario
}

\author{
Mariana Delgado-Jaramillo ${ }^{1}$, Marjorie Machado ${ }^{1}$, Franger J. García ${ }^{1,2}$ \& José Ochoa \\ 1. Departamento de Biología, Facultad Experimental de Ciencias y Tecnología, Universidad de Carabobo, Valencia, \\ Venezuela; marianadelgado13@yahoo.es, fjgarcia4@uc.edu.ve,mmachado3@uc.edu.ve, jochoabat@hotmail.com \\ 2. Grupo de Exploraciones Científicas Minas de Aroa (GECMA - Yaracuy).
}

\author{
Recibido 08-XI-2010. Corregido 18-III-2011. Aceptado 13-IV-2011.
}

\begin{abstract}
Bats (Chiroptera: Mammalia) from Yurubí National Park, Venezuela: taxonomic list and community study. Bats represent a key component in the dynamics of many terrestrial ecosystems, and one of the groups of mammals with the highest levels of diversification in the Neotropics. Here we describe the results of a study of the bat fauna from Yurubí National Park (mountain area in Northern Venezuela), that includes a taxonomic list and the characterization of some community attributes in forested areas. Data was collected from zoological collections and diversified sampling methods from February to July of 2009 in an altitudinal gradient (100-1 500m), with three principal ecological units: semideciduous, evergreen and cloud forests. We recorded 64 species grouped in five families (63\% of the bats known from La Cordillera de la Costa), of which Phyllostomidae was the dominant taxa (42 species; $66 \%$ of total), followed by Vespertilionidae, Molossidae, Emballonuridae and Mormoopidae. The community with the highest taxonomic diversification was found in the lowest elevation range, while the lowest number of species was found at the highest range. Eleven trophic guilds were identified; the insectivorous guild was the richest, whereas the frugivorous was the most abundant. Our results allow us to indicate these forest ecosystems have an appropriate conservation status, taking into account the presence of a relatively high proportion of species from the subfamily Phyllostominae, as well as the presence of other species with conservation priorities. All these aspects, and the fact that this represents a reservoir of the biological diversity of the forest ecosystems of La Cordillera de la Costa, make this protected area of an essential conservation value, in a highly endangered bioregion by neighboring socio-economic growth. Rev. Biol. Trop. 59 (4): 1757-1776. Epub 2011 December 01.
\end{abstract}

Key words: chiroptera, communities, elevation, mammals, Yurubí National Park, Yaracuy, Venezuela.

Diversos estudios han caracterizado la fauna presente en áreas protegidas de la región montañosa del norte de Venezuela, principalmente en la Cordillera de la Costa Central (Bisbal 1993, Manzanilla et al. 1995, Hernández 1997); de ellos, un número importante corresponde a investigaciones mastozoológicas fundamentadas en inventarios taxonómicos o en el diagnóstico de algunas comunidades asociadas con ambientes boscosos (Handley 1976, Ochoa 1986, Fernández-Badillo \& Ulloa 1990, Ochoa et al. 1995, Ochoa et al. 2000, Rivas \& Salcedo 2005, Araujo 2009).
A pesar de los avances existentes en el conocimiento de los mamíferos presentes en la región norte-costera de Venezuela, aún se evidencia la necesidad de evaluar a este grupo faunístico en algunas áreas protegidas como el Parque Nacional Yurubí. Resultados de investigaciones previas llevadas a cabo en localidades cercanas a este parque nacional han sugerido un alto nivel de endemismo local, en el cual se incluye un anfibio (Dendropsophus yaracuyanus; Mijares-Urrutía \& Rivero 2000), un cecílido (Caecilia flavopunctata, Roze \& Solano 1963) y 10 peces (Rodríguez-Olarte et al. 
2005), además de la existencia de algunos táxones endémicos en un contexto regional (e.g., el roedor Ichthyomys pittieri, Linares 1998).

La información expuesta adquiere particular relevancia al considerar que los ecosistemas asociados con el Parque Nacional Yurubí se encuentran sometidos a diferentes presiones antrópicas, tal como ocurre en el resto de la región norte de Venezuela (Huber et al. 1998, Ochoa et al. 1995, OMB 2002). Esto resalta la necesidad de realizar estudios faunísticos orientados a conocer los atributos de las comunidades presentes en aquellas áreas con prioridades de conservación.

La importancia de llevar a cabo estudios comunitarios, radica en que estos son la base para el conocimiento de la estructura, composición y distribución temporal y espacial de las especies en las múltiples zonas de vida y gradientes altitudinales, en donde se describen y definen grupos funcionales (e.g., categorías tróficas, modo de forrajeo o hábitat), con el fin de entender la dinámica, los factores que están determinando la presencia o ausencia de las especies y los patrones de distribución (Soriano 2000). En relación con esto, los resultados de diversos estudios de mamíferos y otros vertebrados han sugerido la disminución de la riqueza de especies como consecuencia de la simplicidad estructural y los gradientes altitudinales, de tal manera que ocurre un remplazo de especies (Graham 1983, 1990, Ochoa \& Gorzula 1992, Ochoa et al. 1993, Rhabek 1995, Patterson et al. 1996, Kalko et al. 1996, Soriano et al. 1999, Ochoa 2000, Soriano 2000, Brown 2001).

Entre los mamíferos conocidos en territorio venezolano, los murciélagos (Orden: Chiroptera) muestran el máximo nivel de diversificación a nivel de especie, y agrupan casi la mitad de los táxones pertenecientes a este grupo de vertebrados (Linares 1998, Ochoa \& Aguilera 2003, Sánchez et al. 2007). Adicionalmente, los murciélagos desempeñan un rol primordial en el mantenimiento y funcionalidad de procesos ecológicos claves, tales como dispersión de semillas, polinización de plantas silvícolas, entre otros (Morton 1989, Kalko et al. 1996, Castro-Arellano et al. 2007). Todos estos aspectos se relacionan con múltiples adaptaciones que tienen los murciélagos para la explotación de una gran variedad de recursos y ambientes, lo que a su vez determina un alto potencial de sensibilidad ante la pérdida o degradación de sus hábitats (Ochoa 2000). Esta última particularidad permite utilizar a este grupo de mamíferos como un excelente indicador del estado de conservación de algunos ecosistemas, sobre todo en aquellos casos donde sus comunidades evidencian un alto grado de complejidad taxonómica y ecológica (Fenton et al. 1992, Wilson et al. 1996, Medellín et al. 2000, Ochoa 2000, Castro-Arellano et al. 2007, Jiménez-Ortega \& Mantilla-Meluk 2008).

Dada la importancia del Parque Nacional Yurubí para la protección de algunos ecosistemas amenazados, principalmente aquellos localizados en áreas circundantes a zonas urbanas del norte de Venezuela, aunado a la poca información taxonómica y ecológica sobre la mastofauna que habita dicha área protegida (particularmente en lo referente a los murciélagos), en este trabajo se presenta una primera caracterización comunitaria de la quiropterofauna asociada a un gradiente altitudinal típico de la Cordillera de la Costa Central de Venezuela.

\section{MATERIALES Y MÉTODOS}

Área de Estudio: El Parque Nacional Yurubí se encuentra ubicado en la Sierra de Aroa (estado Yaracuy), situada en el sector centro-occidental de la Cordillera de la Costa (Fig. 1). Con una superficie de 23 670ha (Bevilacqua et al. 2006) presenta un gradiente altitudinal que varía de 100-1950m e incluye las siguientes unidades ecológicas predominantes: bosques semideciduos y sabanas de montaña (sectores bajos), bosques siempreverdes (sectores medios) y selvas nubladas (sectores más elevados). Según Huber \& Alarcón (1988), la flora regional pertenece a la provincia fitogeográfica del Caribe meridional. El clima es del tipo macrotérmico estacional, con una precipitación anual entre 800-1580mm, 


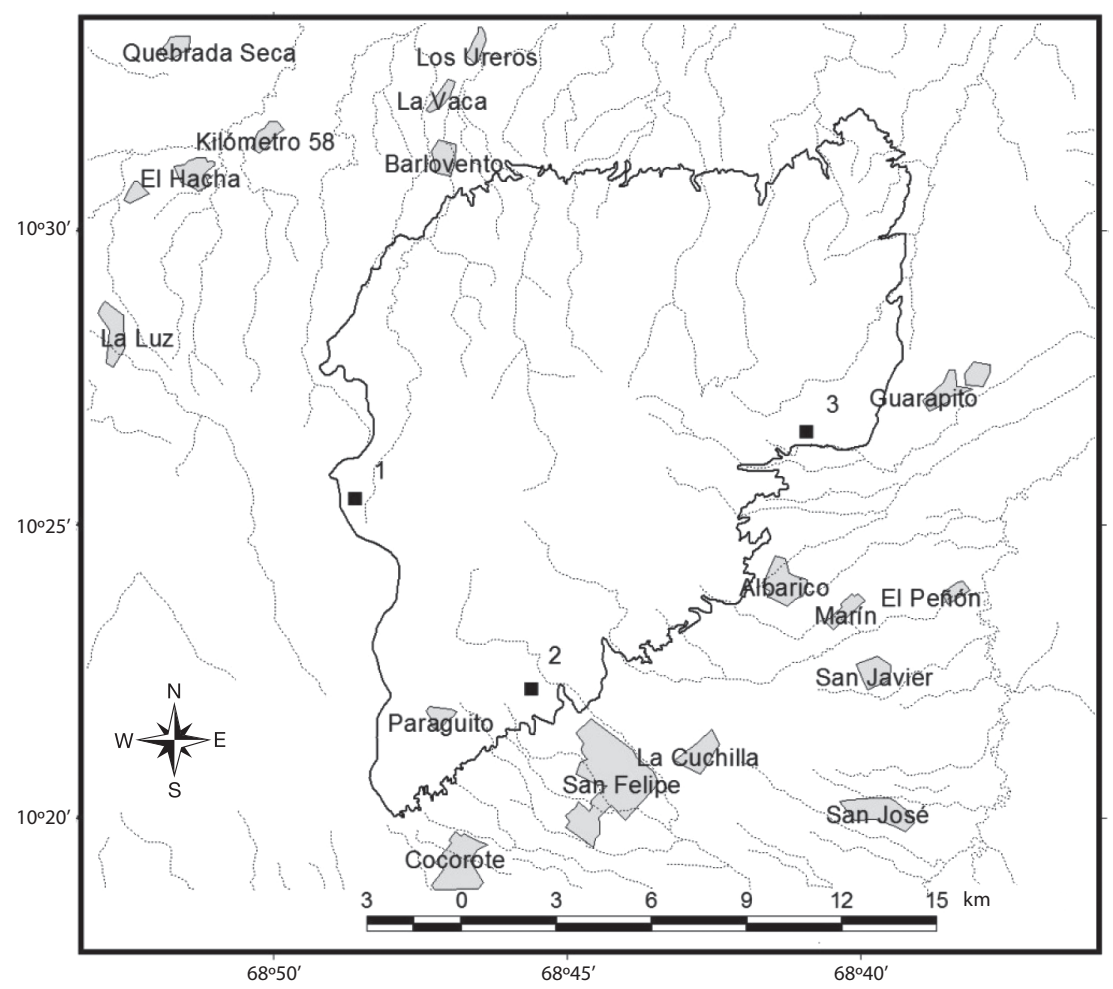

Fig. 1. Parque Nacional Yurubí (Yaracuy, Venezuela) y las localidades evaluadas. 1=El Silencio $\left(10^{\circ} 25^{\prime} \mathrm{N}-68^{\circ} 48^{\prime} \mathrm{O}\right)$; 2=Leonor Bernabó $\left(10^{\circ} 22^{\prime} \mathrm{N}-68^{\circ} 45^{\prime} \mathrm{O}\right)$; 3=Mayorica $\left(10^{\circ} 26^{\prime} \mathrm{N}-68^{\circ} 40^{\prime} \mathrm{O}\right)$.

Fig. 1. Yurubí National Park (Yaracuy, Venezuela) and the localities studied. 1=El Silencio $\left(10^{\circ} 25^{\prime} \mathrm{N}-68^{\circ} 48^{\prime} \mathrm{O}\right)$; $2=\mathrm{Leonor}$ Bernabó $\left(10^{\circ} 22^{\prime} \mathrm{N}-68^{\circ} 45^{\prime} \mathrm{O}\right)$; 3=Mayorica $\left(10^{\circ} 26^{\prime} \mathrm{N}-68^{\circ} 40^{\prime} \mathrm{O}\right)$.

y temperaturas que oscilan entre 10 y $26.5^{\circ} \mathrm{C}$ (Alvarado 2008). A pesar de estar separado de la Cordillera de la Costa por la depresión de Yaracuy, presenta una constitución geológica, faunística y botánica similar a esta cordillera, pero con altos niveles de endemismo (Lentino \& Esclasans 2005, Alvarado 2008).

Para los muestreos relacionados con este estudio se seleccionaron tres localidades (Fig. 1), dentro de un gradiente altitudinal comprendido entre los 100-1500m. A continuación se describen los hábitats evaluados en cada una de estas localidades:

Mayorica: Ubicada entre $100-220 \mathrm{~m}$ $\left(10^{\circ} 26^{\prime} \mathrm{N}-68^{\circ} 40^{\prime} \mathrm{W}\right)$, y en donde se evaluaron dos unidades ecológicas en condición primaria:
1. Bosque Semideciduo: Con árboles de gran altura y con un dosel discontinuo que permite el desarrollo de un sotobosque denso, con los siguientes componentes arbóreos dominantes: Bursera simaruba (Burseraceae), Hura crepitans (Euphorbiaceae), Cedrela sp. (Meliaceae) y Spondias monbin (Anacardiaceae). Adicionalmente, existen representantes de las familias Leguminosaceae, Arecaceae, Selagineceae, Piperaceae, Orchidiaceae y Bromeliaceae (Tillandsia sp.), así como abundantes lianas (Odontocarya sp.) y epifitas (Delascio 1977, Carlos Varela com. pers.).

2. Bosque Siempreverde: Caracterizado por árboles de gran porte y dosel continuo, que incluye Ficus sp. (Moraceae), Gyganthera 
caribensis (Meliaceae) y Brownea grandiceps (Fabaceae), así como un sotobosque con predominio de Rubiaceae, Melastomataceae, Piperaceae (Piper sp.), Lauraceae (Ocotea sp.), Poaceae, Arecaceae, Cyclanthaceae, Acanthaceae, Heliconiaceae (Heliconia psittacorum, Heliconia bihai), Haemodoraceae (Xiphidium caeruleum), Bromeliaceae y Orchidaceae (Epidendrum, Eulophidium) (Delascio 1977, Carlos Varela com. pers.).

Parque Recreacional Leonor Bernabó: Ubicado entre $400-510 \mathrm{~m}\left(10^{\circ} 22^{\prime} \mathrm{N}\right.$ - $\left.68^{\circ} 45^{\prime} \mathrm{W}\right)$, y en donde se evaluaron dos unidades ecológicas:

1. Bosque Semideciduo: Presenta un sector altamente intervenido por deforestaciones, mientras que en el resto de su superficie la vegetación muestra un grado medio de afectación, con un dosel irregular dominado por Bursera simaruba (Burserarecae), Hura crepitans (Euphorbiaceae) y Cedrela sp., (Meliaceae), así como un sotobosque denso con abundante Piper sp., lianas y bejucos.

2. Bosque Siempreverde: También con evidencia de un alto grado de intervención, y la consecuente eliminación de una gran parte de la vegetación original, así como la dominancia de plantas colonizadoras como Cecropia sp. (Moraceae), Solanum sp. (Solanaceae) y Piper sp. (Piperaceae).

El Silencio: Ubicado entre $1414-1460 \mathrm{~m}$ de elevación $\left(10^{\circ} 25^{\prime} \mathrm{N}-68^{\circ} 48^{\prime} \mathrm{W}\right)$, y donde se evaluó una sola unidad ecológica:

1. Bosque Nublado primario: Con un nivel moderado de afectación a lo largo de su periferia; el mismo está caracterizado por un estrato arbustivo y arbóreo desarrollado, con árboles que alcanzan los $30 \mathrm{~m}$ de altura, en donde son muy abundantes las bromelias, gesneriáceas, helechos, orquídeas, así como las familias Lauraceae, Clusiaceae, Meliaceae, Euphorbiaceae,
Lecythidaceae, Marantaceae, Moraceae, Podocarpaceae, Lecythidaceae, Asteraceae, Cyatheaceae, Bombacaceae, Arecaceae y Bromeliaceae.

Adquisición de datos: La información analizada proviene de dos fuentes: datos previos correspondientes a especímenes depositados en colecciones zoológicas, y prospecciones de campo en las localidades anteriormente descritas. En el primer caso, se llevó a cabo una revisión de las bases de datos disponibles en las siguientes instituciones: Museo de la Estación Biológica de Rancho Grande (EBRG), Museo de Zoología de la Universidad de Carabobo (MZUC), Museo de Historia Natural La Salle (MHNLS), Colección de Vertebrados de la Universidad de los Andes (CVULA), Museo de Biología de la Universidad Central de Venezuela (MBUCV), Museo del Instituto de Zoología Agrícola de la Universidad Central de Venezuela (MIZA), American Museum of Natural History (AMNH), National Museum of Natural History (NMNH), Museum of Vertebrate Zoology (MVZ), University of Kansas Biodiversity Institute - Mammal Collection, University of Washington Burke Museum (UWBM) y Utah Museum of Natural History (UMNH); de ellas, sólo en las dos primeras se encontró especímenes recolectados en el Parque Nacional Yurubí.

Las prospecciones de campo fueron llevadas a cabo entre febrero y julio de 2009 , con base en muestreos sistemáticos y diversificados. Se efectuó una salida mensual con una duración de ocho días efectivos cada una para un total de 16 días por piso altitudinal, programadas en los periodos de menor luminosidad lunar. Para las recolectas y registros de murciélagos se utilizaron los siguientes métodos:

- Seis redes de neblina de $18,12,6$ y $2 \mathrm{~m}$ de largo colocadas entre las 18:00-22:00h y de manera interdiaria, entre las 4:00-6:00h, y abarcaron los períodos de mayor actividad de los murciélagos (Simmons \& Voss 1998, Ochoa et al. 2005).

- Una trampa de arpa metálica (AUSTRABAT) de $4 \mathrm{~m}^{2}$ de superficie efectiva, 
colocada en el estrato inferior (1-3.5m) de aquellos sectores boscosos asociados con rutas potenciales de vuelo (Ochoa et al. 2005); la misma era activada durante toda la noche (12h por noche).

- Recorridos diurnos realizados ocasionalmente en cada piso altitudinal, para la búsqueda de refugios, observaciones directas y capturas manuales.

- Registros acústicos computarizados con el software Anabat 6.5, mediante un detector Anabat II conectado con un ZCAIM a una laptop compatible (COMPAQ CONTURA AERO 4/33C). Este equipo permite la recepción, registro y visualización, en tiempo real, de las vocalizaciones de la mayoría de los murciélagos insectívoros que capturan sus presas al vuelo (O'Farrell et al. 1999, Ochoa et al. 2005). Los muestreos fueron realizados estáticamente en los lugares utilizados para la colocación de las redes, en picas o en áreas deforestadas (Simmons \& Voss 1998, Ochoa et al. 2005).

Una parte de los animales capturados fueron identificados y marcados (cortando el pelaje en la parte superior del dorso) para su posterior liberación, mientras que aquellos cuya identidad taxonómica requería de análisis posteriores (e. g., identificaciones de estructuras óseas y dentales en el cráneo) fueron recolectados y se encuentran depositados en el Museo de Zoología de la Universidad de Carabobo (MZUC) y el Museo de la Estación Biológica de Rancho Grande (EBRG). Se siguió el protocolo aprobado por Gannon et al. (2007), para todas las capturas y manipulación de animales en campo. Los criterios taxonómicos considerados siguen a Simmons (2005), Larsen et al. (2007), Gardner (2008) y Lim et al. (2008). Por otra parte, para cada una de las especies se asignó un patrón de distribución geográfica dentro del territorio venezolano, se siguieron dos de las categorías propuestas por Ochoa et al. (1995) y Rivas et al. (2008): Amplia (A), cuando la distribución se extiende al norte y sur del Río Orinoco, y Restringida
(R), cuando la distribución se limita a la región norte del país.

Para el análisis de las estructuras gremiales, se asignaron 11 categorías propuestas por Ochoa (2000), las cuales toman en cuenta las preferencias alimentarias de cada especie y sus tendencias en el uso de los principales estratos de la vegetación: insectívoros aéreos sobre el dosel (IASD), insectívoros aéreos bajo el dosel (IABD), insectívoros de follaje (INFO), insectívoros-carnívoros (INCA), carnívoros (CARN), frugívoros del sotobosque (FRSO), frugívoros del dosel (FRDO), nectarívorosomnívoros (NEOM), nectarívoros-polinívoros (NEPO), omnívoros (OMNI) y hematófagos (HEMA).

El esfuerzo de muestreo fue calculado para cada método por separado en cada una de las localidades. Para las redes de neblina, los resultados se expresan en horas-red-noche (h-red-noche), para la trampa de arpa en horastrampa-noche (h-trampa-noche), los recorridos diurnos en horas-hombre (h-hombre) y los registros acústicos computarizados en horasnoche (h-noche). Los esfuerzos resultantes se muestran en el Cuadro 2. Adicionalmente se calculó el éxito de captura obtenido con mallas y trampa de arpa para cada localidad (Cuadro 2), se utilizó el índice de éxito de captura E, que expresa el cociente del número de individuos capturados con un método, entre su esfuerzo de muestreo (E=N/esfuerzo de muestreo, donde E=Éxito de captura, N=número de individuos capturados).

Las abundancias relativas en el parque e independientemente para los tres pisos altitudinales muestreados (solo se incluyeron los registros provenientes de trampa de arpa y malla que son los mejores estimadores de abundancias) se obtuvieron dividiendo el número de individuos de la especie 1 entre el número total de individuos, multiplicado por 100 para obtener el porcentaje $(P 1=n \mathrm{~s} 1 / \Sigma n$, donde $P 1$ representa la abundancia relativa de la especie1, $n \mathrm{~s} 1$ el número de individuos de la especie1, $\Sigma n$ el número total de individuos). Así mismo, se elaboraron curvas de acumulación de especies para cada piso altitudinal y en los tres sectores 
simultáneamente. Estas curvas fueron realizadas con el programa PAST versión 1.90 (Hammer et al. 2001).

Para medir la diversidad $\alpha$ en cada localidad se determinó el índice de diversidad y equidad de Shannon-Weaner (H') basado en la estructura de la comunidad, con el fin de considerar la distribución proporcional del valor de importancia de cada especie (la abundancia relativa, dominancia, representatividad o equidad) más que la riqueza específica (Magurran 1988). Para la comparación de los pisos altitudinales se utilizó "Shannon Diversity t test" con niveles de significancia $\mathrm{p} \leq 0.05$, para evaluar el nivel de similaridad o disimilaridad de manera significativa (Hammer et al. 2001). En ambos cálculos sólo se tomaron en cuenta los registros provenientes de trampa de arpa y redes de neblina, debido a la falta de valores de abundancia de los registros de museo y acústicos. De tal manera, se cuantificaron las asociaciones de especies o similitudes en la composición de los diferentes pisos altitudinales, utilizando un "Bubble Plot" (SigmaPlot for Windows Versión 10.0) que permite observar la frecuencia de cada especie por elevación.

\section{RESULTADOS}

Composición y riqueza taxonómica: $\mathrm{La}$ quiropterofauna del Parque Nacional Yurubí está constituida por al menos 64 especies (Cuadro 1). Las mismas se encuentran agrupadas en 35 géneros y cinco familias, de las cuales

CUADRO 1

Murciélagos registrados en las tres localidades del Parque Nacional Yurubí, Yaracuy

TABLE 1

Bats recorded in the three localities in the Yurubí National Park, Yaracuy

\begin{tabular}{|c|c|c|c|c|c|c|}
\hline \multirow{2}{*}{ Táxones } & \multicolumn{3}{|c|}{ Localidad de registro } & \multirow{2}{*}{$\begin{array}{l}\text { Método de } \\
\text { registro }\end{array}$} & \multirow{2}{*}{$\begin{array}{l}\text { Distribución } \\
\text { en Venezuela }\end{array}$} & \multirow{2}{*}{ Gremio } \\
\hline & Ma & $\mathrm{Be}$ & $\mathrm{Si}$ & & & \\
\hline \multicolumn{7}{|l|}{ Emballonuridae } \\
\hline Peropteryx kappleri & $\mathrm{x}$ & & & $\mathrm{Mu}$ & A & IABD \\
\hline Saccopteryx bilineata & $\mathrm{x}$ & $\mathrm{x}$ & & $\mathrm{Mu}, \mathrm{Rn}$ & A & IABD \\
\hline Saccopteryx canescens* & $\mathrm{x}$ & & & $\mathrm{Rn}, \mathrm{Ac}$ & A & IABD \\
\hline Saccopteryx leptura* & $\mathrm{x}$ & $\mathrm{x}$ & & Ac & A & IABD \\
\hline \multicolumn{7}{|l|}{ Phyllostomidae } \\
\hline Ametrida centurio** & $\mathrm{x}$ & & & $\mathrm{Mu}$ & A & FRDO \\
\hline Anoura caudifera* & $\mathrm{x}$ & $\mathrm{x}$ & & $\mathrm{Rn}$ & A & NEPO \\
\hline Anoura cultrata & & & $\mathrm{x}$ & $\mathrm{Mu}, \mathrm{Rn}, \mathrm{Ta}$ & $\mathrm{R}$ & NEPO \\
\hline Anoura geoffroyi* & & $\mathrm{x}$ & $\mathrm{x}$ & $\mathrm{Rn}, \mathrm{Ta}$ & A & NEPO \\
\hline Artibeus bogotensis & $\mathrm{x}$ & $\mathrm{x}$ & & $\mathrm{Mu}, \mathrm{Rn}, \mathrm{Ta}$ & A & FRDO \\
\hline Artibeus lituratus & $\mathrm{x}$ & $\mathrm{x}$ & $\mathrm{x}$ & $\mathrm{Mu}, \mathrm{Rn}, \mathrm{Ta}$ & A & FRDO \\
\hline Artibeus planirostris & $\mathrm{x}$ & $\mathrm{x}$ & $\mathrm{x}$ & $\mathrm{Mu}, \mathrm{Rn}$ & A & FRDO \\
\hline Carollia brevicauda & $\mathrm{x}$ & $\mathrm{x}$ & $\mathrm{x}$ & $\mathrm{Mu}, \mathrm{Rn}, \mathrm{Ta}$ & A & FRSO \\
\hline Carollia perspicillata & $\mathrm{x}$ & $\mathrm{x}$ & $\mathrm{x}$ & $\mathrm{Mu}, \mathrm{Rn}, \mathrm{Ta}$ & A & FRSO \\
\hline Chiroderma trinitatum & $\mathrm{x}$ & & & $\mathrm{Mu}$ & A & FRDO \\
\hline Chiroderma villosum & $\mathrm{x}$ & & & $\mathrm{Mu}$ & A & FRDO \\
\hline Choeroniscus godmani* & & $\mathrm{x}$ & & $\mathrm{Rn}$ & A & NEPO \\
\hline Chrotopterus auritus* & $\mathrm{x}$ & & & $\mathrm{Rn}$ & A & CARN \\
\hline Desmodus rotundus & $\mathrm{x}$ & & & $\mathrm{Mu}$ & A & HEMA \\
\hline
\end{tabular}


CUADRO 1 (Continuación)

Murciélagos registrados en las tres localidades del Parque Nacional Yurubí, Yaracuy

TABLE 1 (Continued)

Bats recorded in the three localities in the Yurubí National Park, Yaracuy

\begin{tabular}{|c|c|c|c|c|c|c|}
\hline \multirow{2}{*}{ Táxones } & \multicolumn{3}{|c|}{ Localidad de registro } & \multirow{2}{*}{$\begin{array}{l}\text { Método de } \\
\text { registro }\end{array}$} & \multirow{2}{*}{$\begin{array}{l}\text { Distribución } \\
\text { en Venezuela }\end{array}$} & \multirow{2}{*}{ Gremio } \\
\hline & Ma & $\mathrm{Be}$ & $\mathrm{Si}$ & & & \\
\hline Diphylla ecaudata & $\mathrm{x}$ & & & $\mathrm{Mu}, \mathrm{Rn}$ & A & HEMA \\
\hline Enchisthenes hartii* & & $\mathrm{x}$ & $\mathrm{x}$ & $\mathrm{Rn}, \mathrm{Ta}$ & A & FRDO \\
\hline Glossophaga soricina & $\mathrm{x}$ & $\mathrm{x}$ & $\mathrm{x}$ & $\mathrm{Mu}, \mathrm{Rn}$ & A & NEOM \\
\hline Glyphonycteris sylvestris* & $\mathrm{x}$ & & & $\mathrm{Rn}$ & A & INFO \\
\hline Lampronycteris brachyotis* & $\mathrm{x}$ & & & $\mathrm{Rn}, \mathrm{Ta}$ & A & INFO \\
\hline Lonchorhina aurita & $\mathrm{x}$ & & & $\mathrm{Mu}, \mathrm{Rn}$ & A & INFO \\
\hline Lophostoma brasiliense & $\mathrm{x}$ & & & $\mathrm{Mu}, \mathrm{Rn}$ & A & INFO \\
\hline Lophostoma silvicolum* & $\mathrm{x}$ & $\mathrm{x}$ & & $\mathrm{Rn}$ & A & INFO \\
\hline Micronycteris hirsuta & $\mathrm{x}$ & & & $\mathrm{Mu}, \mathrm{Rn}$ & A & INFO \\
\hline Micronycteris megalotis & $\mathrm{x}$ & & $\mathrm{x}$ & $\mathrm{Mu}, \mathrm{Rn}$ & A & INFO \\
\hline Micronycteris microtis & $\mathrm{x}$ & & $\mathrm{x}$ & $\mathrm{Mu}, \mathrm{Rn}$ & A & INFO \\
\hline Micronycteris minuta & $\mathrm{x}$ & & & $\mathrm{Mu}$ & A & INFO \\
\hline Micronycteris schmidtorum* & $\mathrm{x}$ & & & $\mathrm{Rn}$ & A & INFO \\
\hline Mimon crenulatum & $\mathrm{x}$ & & & $\mathrm{Mu}, \mathrm{Rn}$ & A & INFO \\
\hline Phylloderma stenops & $\mathrm{x}$ & & & $\mathrm{Mu}, \mathrm{Rn}$ & A & OMNI \\
\hline Phyllostomus discolor & $\mathrm{x}$ & & & $\mathrm{Mu}, \mathrm{Rn}$ & A & OMNI \\
\hline Phyllostomus hastatus & $\mathrm{x}$ & & & $\mathrm{Mu}, \mathrm{Rn}$ & A & OMNI \\
\hline Platyrrhinus brachycephalus & $\mathrm{x}$ & & & $\mathrm{Mu}$ & A & FRDO \\
\hline Platyrrhinus helleri & $\mathrm{x}$ & $\mathrm{x}$ & & $\mathrm{Mu}, \mathrm{Rn}, \mathrm{Ta}$ & A & FRDO \\
\hline Platyrrhinus umbratus & & & $\mathrm{x}$ & $\mathrm{Mu}, \mathrm{Rn}$ & $\mathrm{R}$ & FRDO \\
\hline Sphaeronycteris toxophyllum* & $\mathrm{x}$ & & & $\mathrm{Rn}$ & A & FRDO \\
\hline Sturnira erythromos & & & $\mathrm{x}$ & $\mathrm{Mu}$ & A & FRSO \\
\hline Sturnira lilium & $\mathrm{x}$ & $\mathrm{x}$ & $\mathrm{x}$ & $\mathrm{Mu}, \mathrm{Rn}, \mathrm{Ta}$ & A & FRSO \\
\hline Sturnira oporaphilum & & & $\mathrm{x}$ & $\mathrm{Mu}, \mathrm{Rn}, \mathrm{Ta}$ & $\mathrm{R}$ & FRSO \\
\hline Tonatia saurophila & $\mathrm{x}$ & & & $\mathrm{Mu}, \mathrm{Rn}$ & A & INFO \\
\hline Trachops cirrhosus & $\mathrm{x}$ & & & $\mathrm{Mu}$ & A & INCA \\
\hline Uroderma bilobatum & $\mathrm{x}$ & $\mathrm{x}$ & & $\mathrm{Mu}, \mathrm{Rn}$ & A & FRDO \\
\hline Vampyressa thyone & $\mathrm{x}$ & & & $\mathrm{Mu}, \mathrm{Rn}$ & A & FRDO \\
\hline \multicolumn{7}{|l|}{ Mormoopidae } \\
\hline Pteronotus davyi* & & & $\mathrm{x}$ & $\mathrm{Rn}, \mathrm{Ta}, \mathrm{Ac}$ & A & IABD \\
\hline Pteronotus gymnonotus* & & & $\mathrm{x}$ & $\mathrm{Ta}$ & A & IABD \\
\hline Pteronotus parnellii & $\mathrm{x}$ & $\mathrm{x}$ & $\mathrm{x}$ & $\mathrm{Mu}, \mathrm{Rn}, \mathrm{Ta}, \mathrm{Ac}$ & A & IABD \\
\hline Pteronotus personatus & $\mathrm{x}$ & & $\mathrm{x}$ & $\mathrm{Mu}, \mathrm{Rn}, \mathrm{Ta}, \mathrm{Ac}$ & A & IABD \\
\hline \multicolumn{7}{|l|}{ Molossidae } \\
\hline Cynomops greenhalli* & & $\mathrm{x}$ & & $\mathrm{Rn}$ & A & IASD \\
\hline Eumops sp. (prob. auripendulus)* & & $\mathrm{x}$ & & Ac & A & IASD \\
\hline Eumops sp. 1 (prob glaucinus)* & & $\mathrm{x}$ & & Ac & A & IASD \\
\hline Molossus molossus* & $\mathrm{x}$ & $\mathrm{x}$ & & $\mathrm{Rn}, \mathrm{Ac}$ & A & IASD \\
\hline
\end{tabular}


CUADRO 1 (Continuación)

Murciélagos registrados en las tres localidades del Parque Nacional Yurubí, Yaracuy

TABLE 1 (Continued)

Bats recorded in the three localities in the Yurubí National Park, Yaracuy

\begin{tabular}{|c|c|c|c|c|c|c|}
\hline \multirow{2}{*}{ Táxones } & \multicolumn{3}{|c|}{ Localidad de registro } & \multirow{2}{*}{$\begin{array}{l}\text { Método de } \\
\text { registro }\end{array}$} & \multirow{2}{*}{$\begin{array}{l}\text { Distribución } \\
\text { en Venezuela }\end{array}$} & \multirow{2}{*}{ Gremio } \\
\hline & $\mathrm{Ma}$ & $\mathrm{Be}$ & $\mathrm{Si}$ & & & \\
\hline Molossus pretiosus* & & $\mathrm{x}$ & $\mathrm{x}$ & $\mathrm{Rn}, \mathrm{Ac}$ & A & IASD \\
\hline Molossus rufus* & & $\mathrm{x}$ & & Ac & A & IASD \\
\hline \multicolumn{7}{|l|}{ Vespertilionidae } \\
\hline Eptesicus brasiliensis* & $\mathrm{x}$ & & $\mathrm{x}$ & Ac & A & IABD \\
\hline Eptesicus furinalis & $\mathrm{x}$ & $\mathrm{x}$ & $\mathrm{x}$ & $\mathrm{Mu}, \mathrm{Rn}, \mathrm{Ta}, \mathrm{Ac}$ & A & IABD \\
\hline Eptesicus sp. (prob. fuscus)* & & & $\mathrm{x}$ & Ac & $\mathrm{R}$ & IABD \\
\hline Lasiurus sp. (prob. blossevillii)* & & & $\mathrm{x}$ & Ac & A & IABD \\
\hline Lasiurus sp. 1 (prob. ega)* & $\mathrm{x}$ & & $\mathrm{x}$ & Ac & A & IABD \\
\hline Myotis keaysi & $\mathrm{x}$ & & $\mathrm{x}$ & $\mathrm{Mu}, \mathrm{Rn}, \mathrm{Ta}, \mathrm{Ac}$ & $\mathrm{R}$ & IABD \\
\hline Myotis nigricans & $\mathrm{x}$ & $\mathrm{x}$ & $\mathrm{x}$ & $\mathrm{Mu}, \mathrm{Rn}, \mathrm{Ta}, \mathrm{Ac}$ & A & IABD \\
\hline Rhogeessa io & $\mathrm{x}$ & & & $\mathrm{Mu}, \mathrm{Rn}, \mathrm{Ta}, \mathrm{Ac}$ & A & IABD \\
\hline Total & 48 & 25 & 26 & & & \\
\hline
\end{tabular}

(Ma=Mayorica, $\mathbf{B e}=$ Bernabó y $\mathbf{S i}=$ Silencio). $\mathbf{M u}=$ registro de museo; $\mathbf{R n}=$ capturado con red de neblina, $\mathbf{T r}=$ capturado con trampa de arpa y $\mathbf{A c}=$ registro acústico. Los patrones de distribución incluyen: $\mathbf{A}=$ amplia y $\mathbf{R}=$ restringida. Los acrónimos de los gremios se describen en Materiales y Métodos.

* Nuevo registro para el Parque Nacional Yurubí, Yaracuy.

**Recolectada posteriormente a este estudio, en una localidad ubicada a 270m de altitud (Río Guaratarito, Sector Carabobo, Parque Nacional Yurubí, Yaracuy).

Phyllostomidae contiene el $66 \%$ de los táxones inventariados, seguida por Vespertilionidae (13\%), Molossidae (9\%), Emballonuridae (6\%) y Mormoopidae (6\%).

Con un esfuerzo total de 1236 h-red-noche y 440 h-trampa-noche (Cuadro 2) se capturaron
847 individuos (Apéndice) distribuidos en 47 especies durante las 42 noches de muestreo. En la Fig. 2, se muestran las curvas de acumulación de especies por día de muestreo por localidad y para el Parque Nacional de manera general, donde se observa que en ningún caso

\section{CUADRO 2}

Esfuerzos de muestreo y éxitos de captura para el inventario y estudio comunitario de los murciélagos del Parque Nacional Yurubí, Yaracuy

TABLE 2

Sampling efforts and capture success for the inventory and community study of bats from Yurubí National Park, Yaracuy

\begin{tabular}{lcccc}
\multicolumn{1}{c}{ Método } & $\begin{array}{c}\text { Mayorica } \\
(100-220 \mathrm{~m})\end{array}$ & $\begin{array}{c}\text { Bernabó } \\
(400-550 \mathrm{~m})\end{array}$ & $\begin{array}{c}\text { El Silencio } \\
(1414-1460 \mathrm{~m})\end{array}$ & Global del parque \\
Mallas (h-red-noche) & 408 & 408 & 420 & 1236 \\
Trampa de arpa (h-trampa-noche) & 132 & 144 & 164 & 440 \\
Registros acústicos (h-noche) & 4 & 6.5 & 7 & 17.5 \\
Recorridos diurnos (h-hombre) & 28 & 28 & 28 & 84 \\
Éxito de Malla (ind/h-red) & 0.88 & 0.82 & 0.2 & 0.63 \\
Éxito de Arpa (ind/h-trampa) & 0.11 & 0.11 & 0.27 & 0.17 \\
\hline
\end{tabular}



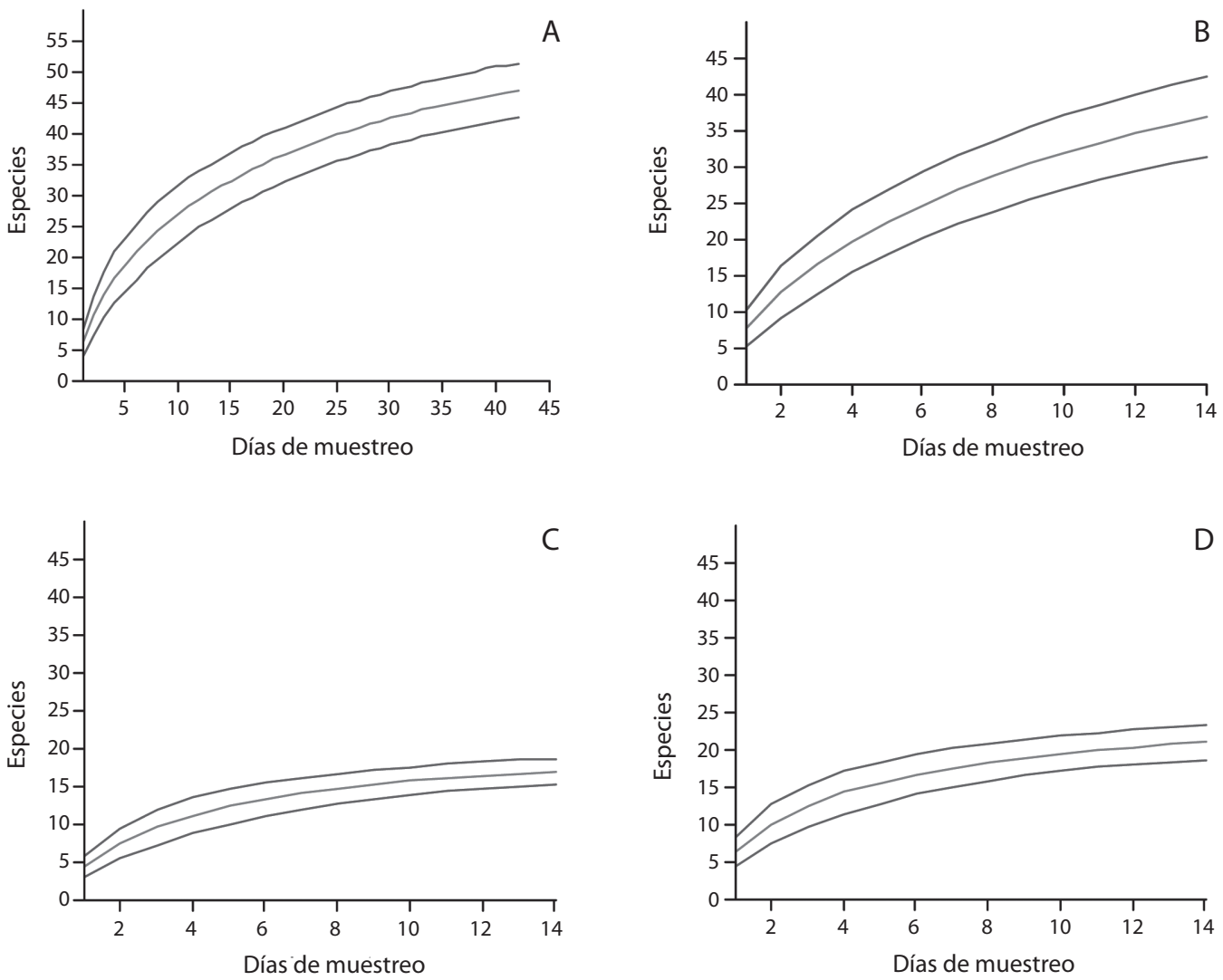

Fig. 2. Curvas de acumulación de especies de murciélagos del Parque Nacional Yurubí durante el periodo de estudio. A: curva global del parque; B, C, D: curvas de las tres localidades evaluadas, B) Mayorica, C) Bernabó y D) El Silencio. Las líneas centrales corresponden a la curva de datos observados, mientras que las líneas laterales corresponden a las curvas de datos esperados con un $95 \%$ de confianza.

Fig. 2. Species accumulation curves of bats from Yurubí National Park during the study period. A: global curve of the park, B, C, D: curves of the three assessed areas, B) Mayorica, C) Bernabó, and D) El Silencio. The center lines correspond to the curve of observed data, while the side lines correspond to the curve of expected data with $95 \%$ confidence.

se alcanzó la asíntota. No obstante, los resultados de este trabajo adicionan 25 especies a la lista de los murciélagos conocidos en el Parque Nacional Yurubí (Cuadro 1) respecto a los datos provenientes de museo, de los cuales ocho fueron registrados exclusivamente mediante detecciones acústicas (Cuadro 1), uno con trampa de arpa (Pteronotus gymnonotus) y el resto con redes.

En este sentido, las mallas capturaron el $80 \%(n=46)$ de las especies registradas en el muestreo y el $91 \%$ de los individuos totales. Mientras que la trampa de arpa capturó el 39\%
(22 spp.) de los táxones y el 9\% de los individuos; por su parte, la acústica registró el $32 \%$ (18 spp.) de los táxones.

De las especies capturadas Carollia perspicillata fue la dominante $(25.7 \%)$, seguida en importancia por Artibeus lituratus, A. planirostris y Sturnira lilium $(14 ; 8.5$ y $7.5 \%$ respectivamente); las cuatro acumularon el $55.7 \%$ de los individuos registrados (Fig. 3.1). El resto de las especies (comunes y raras) presentaron abundancias inferiores a 4\% (Fig. 3.1).

El $92 \%$ de las especies registradas presenta una amplia distribución, mientras que el 
1

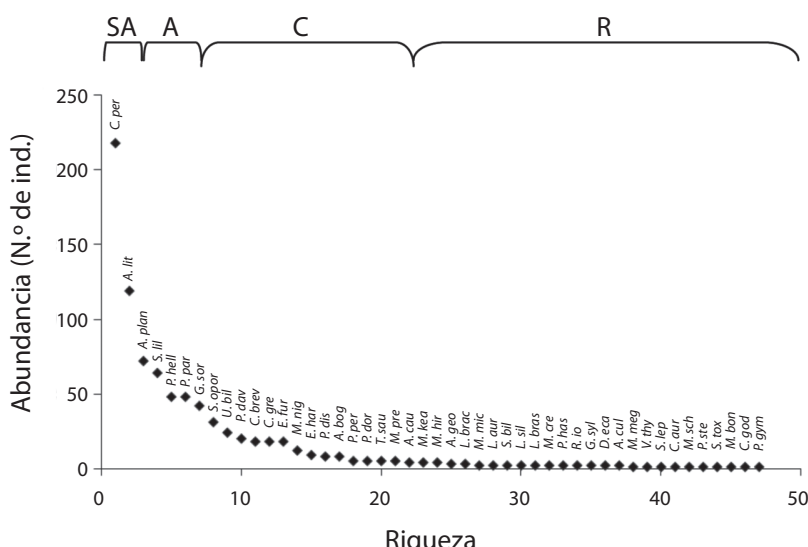

$2 a$
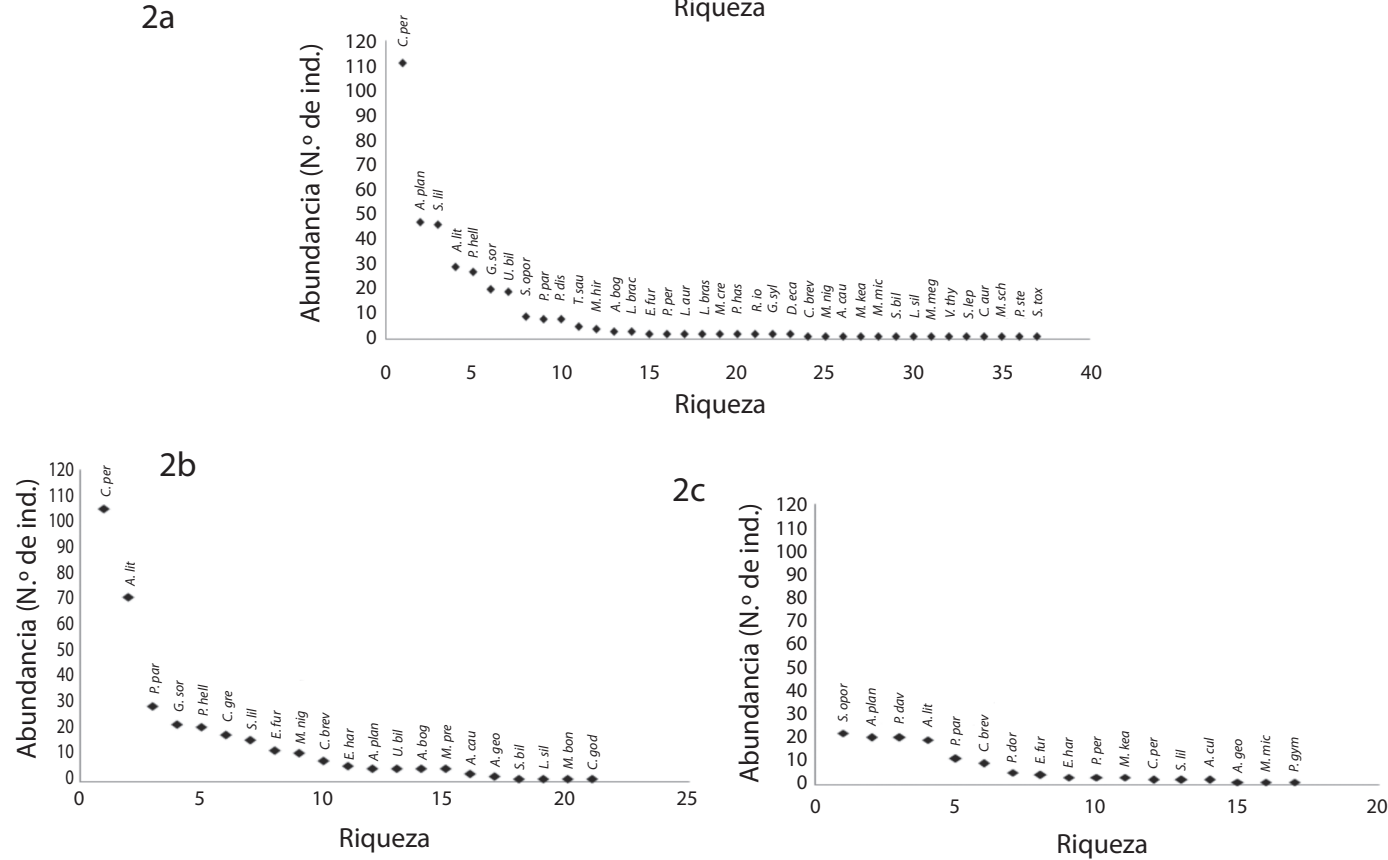

Fig. 3. Curvas de Riqueza-Abundancia de las especies de murciélagos del Parque Nacional Yurubí durante el periodo de estudio. 1) Curva global del parque, 2) Curvas de las tres localidades evaluadas: 2.a) Mayorica, 2.b) Leonord Bernabó, 2.c) El Silencio; SA: superabundantes; A: abundantes; C: comunes; R: raras; Acrónimo de las especies en Apéndice.

Fig. 3. Richness-abundance curves of bats from Yurubí National Park during the study period. 1) Global curve of the park, 2) Curves of the three assessed areas: 2.a) Mayorica, 2.b) Leonord Bernabó, 2.c) El Silencio; SA: superabundant; A: abundant; C: common, R: rare; Acronym for species in Appendix.

$8 \%$ posee una distribución restringida al norte del Orinoco; este último grupo está conformado por Anoura cultrata, Platyrrhinus umbratus, Sturnira oporaphilum, Myotis keaysi y Eptesicus fuscus.
Análisis comunitario: Con respecto a la distribución altitudinal en el área de estudio, la riqueza taxonómica disminuyó a medida que se asciende en el gradiente altitudinal. El valor máximo de diversidad $\left(\mathrm{H}^{\prime}=2.498\right)$ 
correspondió a Mayorica (piso inferior 100$220 \mathrm{~m}$ ) con una riqueza de 48 especies, de las cuales 18 (Fig. 4; Cuadro 1), se registraron exclusivamente en este piso altitudinal $(38.3 \%$ de los murciélagos inventariados en toda el área de estudio). Cabe destacar la alta representatividad a nivel de especies de la subfamilia Phyllostominae en esta localidad (17 spp.). A nivel de individuos capturados, en este piso dominaron las especies Carollia perspicillata con 111 individuos que representó el 30\% del total de individuos para la localidad, seguido de Artibeus planirostris con 13\% $(\mathrm{n}=47)$ y Sturnira lilium con $12 \%$ (Fig. 3.2a).

La comunidad asociada con el piso medio (400-550m) evidenció la menor diversidad $\left(H^{\prime}=2.295\right)$, con una riqueza de 25 especies; sólo seis especies fueron inventariadas exclusivamente en este piso (Fig. 4; Cuadro 1). Esta comunidad estuvo dominada por Carollia perspicillata con el $30.1 \%$ de los individuos capturados ( $\mathrm{n}=105)$, en segundo lugar Artibeus lituratus con $20.4 \%(\mathrm{n}=71)$, seguido de Pteronotus parnellii con $8.3 \%$ (Fig. 3.2b). Los valores anteriores condicionan el menor grado de similaridad entre los pisos altitudinales bajo y medio, siendo el único caso donde se encontraron diferencias significativas $(\mathrm{t}=2.031, \mathrm{p}<0.05)$, mientras que entre el piso bajo y superior no se encontraron diferencias significativas, aunque la similaridad es baja ( $\mathrm{t}=1.385 ; \mathrm{p}=0.167)$.

La comunidad del bosque nublado de El Silencio (1 440-1 460m) mostró un valor intermedio de diversidad $\left(H^{\prime}=2.371\right)$ y el mayor

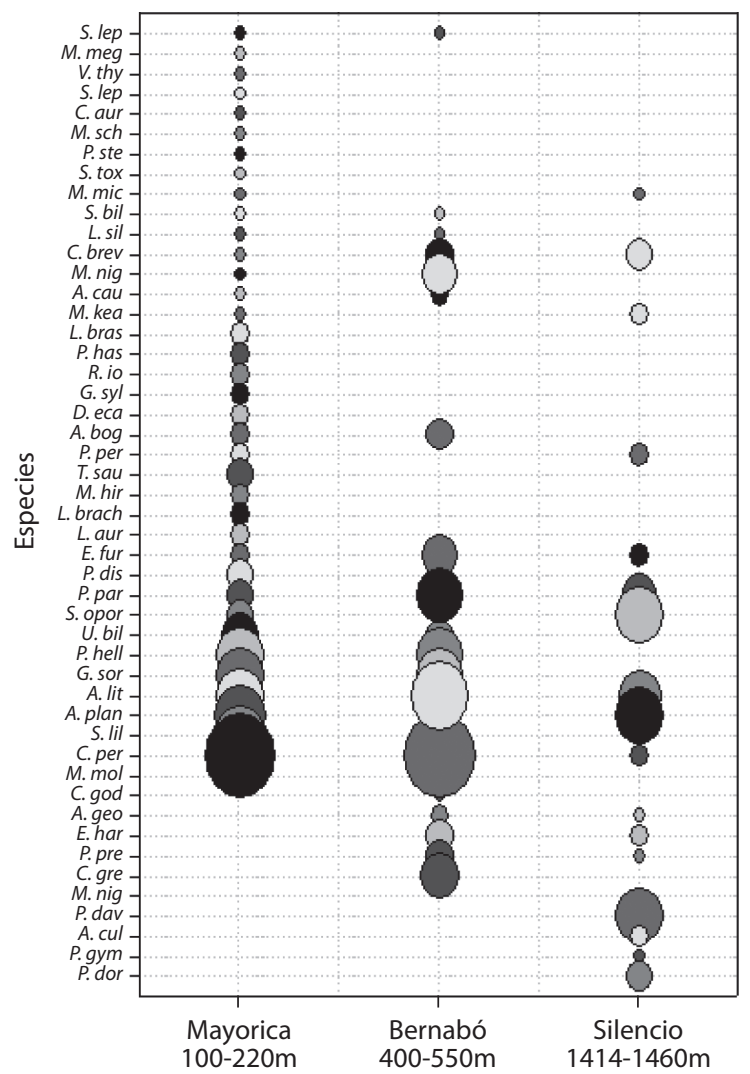

Fig. 4. Distribución y abundancia relativa de las especies de murciélagos en el gradiente altitudinal evaluado en el Parque Nacional Yurubí, Yaracuy. Acrónimo de las especies en Apéndice.

Fig. 4. Distribution and relative abundance of bat species in an altitudinal gradient evaluated for the Yurubí National Park, Yaracuy. Acronym for species in Appendix. 
valor de similaridad con el piso medio $(\mathrm{t}=$ $0.433 ; \mathrm{p}=0.665$ ), con una riqueza de 26 especies, seis de ellas restringidas a este sector (Fig. 4; Cuadro 1). Por su parte, en esta comunidad dominaron: Sturnira oporaphilum con el $17.2 \%(\mathrm{n}=22)$, Artibeus planirostris y Pteronotus davyi ambos con $15.6 \%$ cada uno $(\mathrm{n}=20)$ $\mathrm{y}$ finalmente con $14.8 \%(\mathrm{n}=19)$ A. lituratus (Fig. 3.2c).

En el sector El Silencio se obtuvo el valor máximo de éxito de captura $(0.27 \mathrm{ind} / \mathrm{h})$ con trampa de arpa (Cuadro 2), lo que correspondió al 59\% de las especies capturadas en este piso altitudinal; por el contrario, en esta misma localidad se observa el menor éxito de captura $(0.2$ ind/h-malla) con mallas de neblina (Cuadro 2). En las localidades de Mayorica y Bernabó se concentró el mayor éxito de captura mediante este último método con 0.88 y $0.82 \mathrm{ind} / \mathrm{h}$ malla, respectivamente (Cuadro 2), mientras que con trampas de arpa se registró un valor similar entre ambas localidades $(0.11 \mathrm{ind} / \mathrm{h}$ trampa) y menor al bosque nublado.

Con respecto a la estructura gremial de manera general, en el Parque Nacional Yurubí se observó una dominancia del hábito insectívoro con casi el $54 \%$ del total de especies registradas $(n=34)$. En segundo lugar, se encuentra el hábito frugívoro que acumuló el $28 \%$ de las especies $(n=18)$, mientras que el resto de los gremios están representados por menos de $7 \%$ cada uno.

Al hacer la discriminación por piso, la comunidad de Mayorica presentó la mayor complejidad estructural, incluyendo los 11 gremios propuestos (Fig. 5). Los INFO e IABD dominaron con 12 especies ( $25 \%$ ) en cada caso, seguidos por FRDO (10 especies- 20.8\%), el resto de los gremios presentaron abundancias relativas menores al 10\%. En Bernabó se evidenció una simplificación de la estructura gremial con la presencia de solo siete gremios (Fig. 5), y los mejores representados los IASD y FRDO con séis especies (24\%) cada uno, seguido de los IABD (5 especies- 20\%), y por último los FRSO y NEPO con tres especies en cada caso (Fig. 5). De igual manera, en El Silencio se registraron siete gremios (Fig. 5),

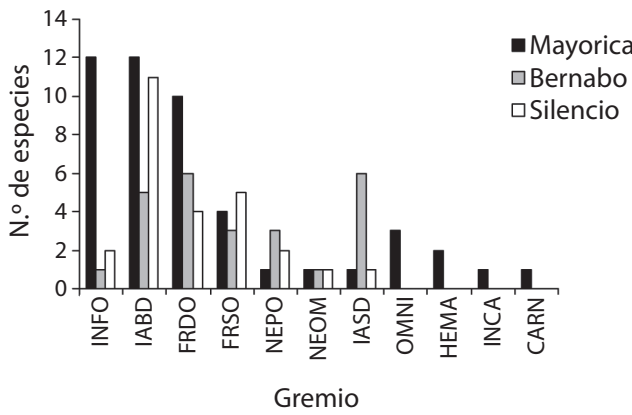

Fig. 5. Representatividad de la estructura gremial de las comunidades de murciélagos del Parque Nacional Yurubí, Yaracuy.

Fig. 5. Representativeness of the guild structure of bat communities from Yurubí National Park, Yaracuy.

siendo IABD el dominante (con 11 especies$42.3 \%$ ), seguido por FRSO (5 especies- 19.2\%) y FRDO (4 especies- 15.4\%).

\section{DISCUSIÓN}

Los resultados obtenidos ubican al Parque Nacional Yurubí entre los sectores de la Cordillera de la Costa con las mayores riquezas de murciélagos conocidas (Ochoa 1986, Bisbal 1993, MARN 1995, Ochoa et al. 1995, Ochoa et al. 2000, Rivas-Rojas 2005, Rivas \& Salcedo 2005, García et al. 2007, Machado 2007, Oria 2007, Araujo 2009), al tener representado el $38 \%$ de la riqueza taxonómica señalada en territorio venezolano (Gardner 2008, Sánchez et al. 2007) y el $63 \%$ del valor conocido para la Cordillera Central de la Costa (Linares 1998).

Con este estudio se incrementó el listado de especies para esta localidad en un $39 \%$. Adicionalmente, se amplía la distribución de la especie Micronycteris schmidtorum, la cual es señalada por primera vez para la Cordillera de la Costa, mientras que Lampronycteris brachyotis era desconocido hasta ahora en el estado Yaracuy y Glyphonycteris sylvestris constituye el segundo registro para el estado (Ojasti \& Mondolfi 1968, Handley 1976, Ochoa 1986, Fernández-Badillo \& Ulloa 1990, Bisbal 1993, Ochoa et al. 1995, Linares 1998, 
Rivas \& Salcedo 2005, García et al. 2007, Machado 2007, Oria 2007, SIMCOZ 2008, Araujo 2009).

De las especies encontradas de distribución restringida al norte de Venezuela, $S$. oporaphilum y $P$. umbratus se encuentran en todas las bioregiones definidas para el país (Linares 1998), con excepción de la cuenca del Lago de Maracaibo en ambos casos y los Llanos para la segunda. Por su parte, A. cultrata, M. keaysi y E. fuscus únicamente han sido señaladas en los Andes y la Cordillera de la Costa Central, el Sistema Coriano se incluye para la última de ellas (Linares 1998), lo que representa un aspecto clave desde el punto de vista de conservación.

Respecto a las técnicas de registro es de resaltar que el uso de acústica incrementó en un $15 \%$ la lista obtenida con trampa y mallas. En este sentido, la acústica constituyó una herramienta clave en el registro de representantes de la familia Molossidae, Vespertilionidae y Emballonuridae, mientras que la trampa de arpa resultó muy eficiente para la captura de algunas especies no tan susceptibles a las mallas principalmente insectívoros aéreos bajo dosel (Vespertilionidae y Mormoopidae). En contraposición, las mallas de neblina fueron muy eficientes en la captura de murciélagos con poca capacidad de ecolocalización (principalmente Phyllostomidae), y aporta gran porcentaje de la información obtenida para cada ensamblaje en concordancia con lo descrito por muchos autores (Kalko et al. 1996, O'Farrell \& Gannon 1999).

A partir de los resultados obtenidos en campo en concordancia con los planteamientos de investigadores que han utilizado todas las técnicas de registro, se hace evidente que todos los métodos de muestreo de murciélagos presentan un sesgo particular, es por ello que todos los resultados sugieren que la combinación de las tres técnicas de registro es lo más apropiado para obtener los mejores resultados al momento de evaluar las comunidades de murciélagos (O'Farrell et al. 1999, O'Farrell \& Gannon 1999, Ochoa et al. 2000)
Hasta el momento, en el área de estudio no se han registrado 21 especies que han sido señaladas para la Sierra de Aroa y otras localidades de la Cordillera Central por diversos autores (Ojasti \& Mondolfi 1968, Handley 1976, Fernández-Badillo \& Ulloa 1990, Rivas \& Salcedo 2005, García et al. 2007, Machado 2007), sin embargo su presencia se considera bastante probable (e. g., Peropteryx macrotis, Myotis oxyotus, Trinycterys nicefori, Vampyrum spectrum y Natalus tumidirostris). La ausencia de estas especies, aunado a la falta de un punto de inflexión en las curvas de acumulación y a la presencia de un número importante de táxones representados por un solo individuo, sugieren la necesidad de continuar los esfuerzos orientados a muestreos diversificados (que minimicen el sesgo particular de cada método de registro), con escalas temporales y espaciales más amplias (Kalko et al. 1996, Simmons \& Voss 1998, O'Farrell 1999, Voss et al. 2001, Sampaio et al. 2003).

De manera general, la mayor riqueza taxonómica y alta complejidad en la estructura gremial encontrada en Mayorica (piso inferior) estaría condicionada por la presencia de bosques primarios, continuos y estructuralmente diversificados, lo que a su vez determina una condición óptima en la oferta, disponibilidad y distribución de recursos (Klopher \& MacArthur 1961, Graham 1983, Ochoa et al. 1988, Graham 1990, Kalko et al. 1996, Soriano et al. 1999, Ochoa 2000, Soriano \& Ochoa 2001, Ochoa et al. 2005); esto se ve reflejado en la dominancia de gremios asociados con el consumo de insectos y frutas, los cuales mostraron en esta localidad los mayores niveles de diversidad y riqueza.

La elevada representatividad de insectívoros de follaje (subfamilia Phyllostominae) en este piso altitudinal inferior $(85 \%$ de las especies registradas para la Cordillera de la Costa), corrobora lo señalado por otros autores en lo que respecta al incremento en la frecuencia de aparición de este grupo taxonómico en ambientes boscosos conforme aumenta su nivel de conservación en virtud de sus hábitos alimentarios y de refugio especialista (Fenton 
et al. 1992, Wilson et al. 1996, Medellín et al. 2000, Ochoa 2000, Ochoa \& Soriano 2001, Faria 2006, Presley et al. 2008). Por otra parte, la baja aparición de los nectarívoros en Mayorica respecto a las otras localidades, concuerda con lo señalado por algunos autores respecto al incremento en la probabilidad de encuentro de los representantes de este gremio en hábitats boscosos secundarios (Fenton et al. 1992, Ochoa 2000, Willig et al. 2007).

Una condición opuesta se encontró en Bernabó (piso medio), aún cuando presenta las mismas unidades ecológicas de Mayorica, las cuales además fueron muestreadas con esfuerzos equivalentes. Este patrón comunitario estaría determinado por la mayor simplificación de los ecosistemas boscosos en esta localidad como resultado de intervenciones antrópicas (Soriano \& Ochoa 2001, Ochoa et al. 2005, Willig et al. 2007). Entre otros aspectos, esto se traduce en una disminución en la disponibilidad de recursos alimentarios típicos de bosques primarios, la reducción y/o eliminación de estratos de movilidad, la modificación en las condiciones microclimáticas del sotobosque y la desaparición de refugios potenciales asociados con la presencia de árboles de gran porte (Soriano \& Ochoa 2001, Ochoa et al. 2005, Willig et al. 2007, Presley et al. 2008).

En el caso de Bernabó, la abundancia de frugívoros de sotobosque (principalmente Carollia sp.) estaría indicando la presencia de hábitats secundarios, debido a que una parte importante de la dieta de estos murciélagos proviene de plantas colonizadoras como Solanum sp., Piper sp. y Cecropia sp. (Medellín et al. 2000, Ochoa et al. 2005, Faria 2006, Griscom et al. 2006, Meyer 2007). Asimismo es de resaltar que en esta localidad se presentaron las únicas capturas y en general el mayor número de registros acústicos de insectívoros aéreos sobre dosel (principalmente Molossidae), lo cual podría explicarse por la existencia de amplias áreas abiertas que facilitan su detección y la presencia de construcciones (galpones, casas y puentes) utilizadas como refugios preferenciales por este grupo gremial (Jennings et al. 2000).
La similaridad encontrada entre la quiropterofauna de Bernabó y El Silencio en términos de su riqueza, diversidad y simplicidad de la estructura gremial (a pesar de sus diferencias altitudinales), podría estar determinada por las influencias que ejercen las condiciones climáticas de tierras altas sobre la comunidad de esta última localidad. Esto es apoyado por diversos estudios realizados en ambientes tropicales, cuyos resultados sugieren que tanto la pérdida de hábitats originales, como las severas presiones térmicas que imponen las tierras altas influye negativamente en la presencia, abundancia y persistencia de muchas especies. Lo que condiciona en ambos casos comunidades menos complejas que las encontradas en tierras bajas primarias (Graham 1990, Ochoa et al. 1993, Kalko et al. 1996, Laurance \& Bierregaard 1997, Soriano et al. 1999, Soriano 2000).

La comunidad de murciélagos de El Silencio, a pesar de presentar una estructura gremial similar a la del Bernabó, posee una constitución taxonómica de especies frugívoras y nectarívoras típicas de tierras altas del norte de Venezuela (e. g., Platyrrhinus umbratus y Anoura cultrata); por otro lado, resalta además la ausencia de la familia Emballonuridae, cuya distribución, para la mayoría de las especies neotropicales se restringe a tierras bajas y medias (Graham 1983, Soriano 1983, Graham 1990, Ochoa \& Gorzula 1992, Ochoa et al. 1993, Soriano 2000).

Finalmente, conviene resaltar que la presencia en el Parque Nacional Yurubí de cinco especies de murciélagos con distribuciones restringidas al norte del Orinoco y de cuatro especies incluidas en el libro Rojo de la Fauna Venezolana (Choeroniscus godmani, Diphylla ecaudata, Glyphonycteris sylvestris y Platyrrhinus umbratus) (Rodríguez \& Rojas-Suárez 2008), además de la existencia de un alto grado de endemismo y la alta representatividad de la subfamilia Phyllostominae en algunos sectores, le confieren a esta área protegida un alto valor desde el punto de vista de protección, en virtud de su importancia como reservorio de la diversidad biológica que caracteriza a la Cordillera de la Costa. 


\section{AGRADECIMIENTOS}

Al Departamento de Biología de la Facultad Experimental de Ciencias y Tecnología de la Universidad de Carabobo, por el apoyo logístico e institucional. Al Programa Iniciativa de Especies Amenazadas (IEA) de PROVITA por el financiamiento adjudicado a este trabajo. A Héctor Silva, Karen López, Luis Aular, Frank Espinoza, Yoiber Mujica, Jorge Giménez $\dagger$, Edward Camargo, Hilda Siliet, Carlos Varela y Eliécer Gutiérrez. A Javier Sánchez y Francisco Bisbal por la atención dispensada en el Museo de la Estación Biológica de Rancho Grande (MEBRG) y por su colaboración en las identificaciones taxonómicas. Al Ministerio del Poder Popular para el Ambiente e Instituto Nacional de Parques (INPARQUES) por los permisos correspondientes. Al Destacamento de la Guardia Nacional y al personal de la planta de tratamiento Aguas de Yaracuy ubicados en el Parque Recreacional Leonor Bernabó, por sus atenciones y colaboración.

\section{RESUMEN}

Los murciélagos constituyen el grupo de mamíferos más diversificado en la región neotropical y representan un componente clave en la dinámica de muchos ecosistemas terrestres. En este trabajo se describen los resultados de un estudio sobre la quiropterofauna del Parque Nacional Yurubí (ubicado en la zona montañosa del norte de Venezuela), se incluye un listado taxonómico y la caracterización de algunos atributos comunitarios en ambientes predominantemente boscosos. Los datos evaluados fueron obtenidos mediante la revisión de colecciones zoológicas y muestreos diversificados realizados entre febrero y julio del 2009, en un gradiente altitudinal (100-1 500m), que integra a tres unidades ecológicas: bosque semideciduo, bosque siempreverde y selva nublada. Se registraron 64 especies agrupadas en cinco familias, lo que constituye el $63 \%$ de los murciélagos conocidos en la Cordillera de la Costa. La familia Phyllostomidae fue dominante (42 especies; $66 \%$ del total), seguida por Vespertilionidae, Molossidae, Emballonuridae y Mormoopidae. La comunidad presente a baja elevación fue la más diversificada en términos taxonómicos, mientras que la del piso superior evidenció el menor grado de diversificación. Se identificaron 11 categorías tróficas, con dominancia de los insectívoros en cuanto a la riqueza taxonómica y de los frugívoros en lo que respecta a la abundancia. Nuestros resultados permiten inferir, en un contexto general, que los ecosistemas boscosos del área de estudio muestran un buen estado de conservación, en virtud de la presencia de una proporción relativamente alta de especies pertenecientes a la subfamilia Phyllostominae, cuyos componentes han sido considerados por otros autores como indicadores del grado de conservación de hábitats boscosos primarios, así como la presencia de otras especies con prioridades de conservación. Todos estos aspectos, le confieren a esta área protegida un alto valor proteccionista, en virtud de su importancia como reservorio de la diversidad biológica que tipifica los ecosistemas boscosos de la Cordillera de la Costa, una bio-región altamente amenazada como consecuencia de un elevado crecimiento socio-económico.

Palabras clave: chiroptera, comunidades, elevación, mamíferos, Parque Nacional Yurubí, Yaracuy, Venezuela.

\section{REFERENCIAS}

Alvarado, H. 2008. Aspectos estructurales y florísticos de cuatro bosques ribereños de la cuenca del río Aroa, estado Yaracuy, Venezuela. Act. Bot. Venez. 31: 273-290.

Araujo, D. 2009. Composición de las comunidades de murciélagos (Mammalia: Chiroptera) del Monumento Natural Pico Codazzi (estados Aragua, Miranda y Vargas). Tesis de licenciatura, Universidad de Carabobo, Valencia, Venezuela.

Bevilacqua, M., L. Cárdenas \& D. Medina. 2006. Las Áreas protegidas en Venezuela: Diagnóstico de su condición 1993/2004. Fundación Polar, ACOANA, IUCN, Caracas, Venezuela.

Bisbal, F. 1993. Inventario preliminar de la fauna de la cuenca del rió Morón, Estado Carabobo, Venezuela. Acta Cient. Venez. 44: 365-382.

Brown, J. 2001. Mammals on mountainsides: elevational patterns of diversity. Global Ecol. Biogeogr. 10: 101-109.

Castro-Arellano, I., S. Presley, L. Saldanha, M. Willig \& J. Wunderle. 2007. Effects of reduced impact logging, on bat biodiversity in terra firme forest of lowland Amazonia. Biol. Conservat. 138: 269-285.

Delascio, F. 1977. Notas sobre la flora del Yurubí, Estado Yaracuy, Venezuela. Mem. Soc. Cien. Nat. La Salle 37: 265-281.

Faria, D. 2006. Phyllostomid bats of a fragmented landscape in the North-Eastern atlantic forest, Brazil. J. Trop. Ecol. 22: 531-542. 
Fenton, M., L. Acharya, L. Audet, M. Hickey, C. Merriman, M. Obrist, D. Syme \& B. Adkins. 1992. Phyllostomid bats (Chiroptera: Phyllostomidae) as indidicators of habitat disruption in the Neotropics. Biotropica 24: 440-446.

Fernández-Badillo, A. \& G. Ulloa. 1990. Fauna del Parque Nacional Henri Pittier, Venezuela: composición y diversidad de la mastofauna. Acta Cient. Venez. 41: 50-63.

Gannon, W. \& R. Sikes. The animal care and use committee of the American Society of Mammalogists. 2007. Guidelines of the American Society of Mammalogists for the use of wild mammals in research. J. mammal. 88: 809-823.

García, F., L. Aular, E. Camargo \& Y. Mujica. 2007. Murciélagos de la Sierra de Aroa, Estado Yaracuy. Libro de Resúmenes del VII Congreso Venezolano de Ecología. La sociedad es parte del ecosistema. Ciudad Guayana, Venezuela.

Gardner, A. 2008. Mammals of South America, Volumen 1: Marsupials, Xenarthrans, Shrews and Bats. The University of Chicago, Chicago, EEUU.

Graham, G. 1983. Changes in bat species diversity along an elevational gradient up the peruvian Andes. J. Mammal. 64: 559-571.

Graham, G. 1990. Bats versus birds: comparisons among peruvian volant vertebrate faunas along an elevational gradient. J. Biogeogr. 17: 657-668.

Griscom, H., E. Kalko \& M. Ashton. 2006. Frugivory by small vertebrates within a deforested, dry tropical region of Central America. Biotropica 39: 278-282.

Hammer, O., D. Harper \& P. Ryan. 2001. PAST: Paleontological sofware package for education and data analysis. Paleontología Electrónica: 9. (Disponible en línea: http://folk.uio.no/ohammer/past).

Handley, C. 1976. Mammals of the Smithsonian Venezuelan Project. Brigham Young Univ. Sci. Bull. Biol. Ser. 20: 1-91.

Hernández, O. 1997. Estudio comparativo de las comunidades de vertebrados terrestres (epigeos), de dos bosques ombrófilos submontanos y montanos siempreverde de la Cordillera de la Costa, Aragua, Venezuela. Tesis de licenciatura, Universidad Central de Venezuela, Maracay, Venezuela.

Huber, O. \& C. Alarcón. 1988. Mapa de vegetación de Venezuela. MARN-BIOMA, Caracas, Venezuela.
Huber, O., R. Duno, R. Riina, F. Stauffer, L. Papaterra, A. Jiménez, S. Llamozas \& G. Orsini. 1998. Estado actual del conocimiento de la flora en Venezuela. Documentos Técnicos de la Estrategia Nacional de Diversidad Biológica, Caracas, Venezuela.

Jennings, J., T. Best, J. Rainey \& E. Burnett. 2000. Molossus pretiosus. Mamm. Species 635: 1-3.

Jiménez-Ortega, A. \& H. Mantilla-Meluk. 2008. El papel de la tala selectiva en la conservación de bosques neotropicales y la utilidad de los murciélagos como bioindicadores de disturbio. Revista Institucional Universidad Tecnológica del Chocó: Investigación. Biodiversidad y Desarrollo 27: 100-108.

Kalko, E., C. Handley \& D. Handley. 1996. Organization, diversity, and long-term dynamics of a neotropical bat community, p. 503-553. In M. Cody \& J. Smallwood. Long-term studies of vertebrate communities. Academic, Nueva York, EEUU.

Klopher, P. \& R. MacArthur. 1961. On the causes of tropical species diversity: niche overlap. Am. Nat. 95: 223-226.

Larsen, P., S. Hoofer, M. Bozeman, S. Pedersen, H. Genoways, C. Phillips, D. Pumo \& R. Baker. 2007. Phylogenetics and phylogeography of the Artibeus jamaicensis complex based on cytochrome- $b$ DNA sequences. J. Mammal. 88: 712-727.

Laurance, W. \& R. Bierregaard. 1997. Tropical forest remnants: ecology, management, and conservation of fragmented communities. University of Chicago, Chicago, EEUU.

Lentino, M. \& D. Esclasans. 2005. Áreas importantes para la conservación de las aves en Venezuela, p. 621-730. In BirdLife International y Conservation International. Áreas importantes para la conservación de las aves en los Andes tropicales: sitios prioritarios para la conservación de la biodiversidad. BirdLife International, Quito, Ecuador.

Lim, B., M. Engstrom, J. Patton \& J. Bickham. 2008. Systematic review of small fruit-eating bats (Artibeus) from the Guianas, and a re-evaluation of A. glaucus bogotensis. Acta Chiropterol. 10: 243-256.

Linares, O. 1998. Mamíferos de Venezuela. Sociedad Conservacionista Audobon de Venezuela, Caracas, Venezuela.

Magurran, A. 1988. Ecological diversity and its measurement. Princeton University, Nueva Jersey, EEUU.

Machado, M. 2007. Murciélagos del extremo occidental del Macizo de Nirgua, Estado Yaracuy, Venezuela. 
Libro de Resúmenes del VII Congreso Venezolano de Ecología. La sociedad es parte del ecosistema. Ciudad Guayana, Venezuela.

Manzanilla, J., A. Fernández-Badillo, E. La Marca \& R. Visval. 1995. Fauna del Parque Nacional Henri Pittier, Venezuela: composición y distribución de los anfibios. Acta Cient. Venez. 46: 294-302.

MARN (Ministerio del Ambiente y de los Recursos Naturales). 1995. Inventario preliminar de la fauna de la sierra sur de Bobare (Sector el Jaguar). Estados Lara y Falcón. Informe Técnico, Maracay, Venezuela.

Medellín, R., M. Equihuana \& M. Amin. 2000. Bat diversity and abundance as indicators of disturbance in Neotropical rainforest. Biol. Conservat. 14: 1666-1675.

Meyer, C. 2007. Effects of rainforest fragmentation on neotropical bats Land-bridge islands as a model system. Tesis de doctorado, Universidad de Ulm, Ulm, Alemania.

Mijares-Urrutía, A. \& R. Rivero. 2000. A new treefrog from the Sierra de Aroa, Northern Venezuela. J. Herpetol. 34: 80-84.

Morton, P. 1989. Murciélagos tropicales americanos. El Fondo Mundial Para La Naturaleza, Washington, EEUU.

Ochoa, J. 1986. Inventario preliminar de la mastofauna del tramo occidental de la Cordillera de la Costa. Informe Técnico. MARN-DGSIIA, Maracay, Venezuela.

Ochoa, J., J. Sánchez, M. Bevilacqua \& R. Rivero. 1988. Inventario de los mamíferos de la Reserva Forestal de Ticoporo y la Serranía de los Pijiguaos, Venezuela. Acta Cient. Venez. 39: 269-280.

Ochoa, J. \& S. Gorzula. 1992. Los mamíferos del macizo de Chimantá con algunos comentarios sobre las comunidades de las cumbres tepuyanas, p. 295-302. In O. Huber. El macizo del Chimantá, Escudo de Guayana, Venezuela: Un ensayo Ecológico Tepuyano. Oscar Todtmann, Caracas, Venezuela.

Ochoa, J., C. Molina \& S. Giner. 1993. Inventario y estudio comunitario de los mamíferos del Parque Nacional Canaima, con una lista de las especies registradas para la Guayana venezolana. Acta Cient. Venez. 44: 245-262.

Ochoa, J., M. Aguilera \& P. Soriano. 1995. Los mamíferos del Parque Nacional Guatopo (Venezuela): lista actualizada y estudio comunitario. Acta Cient. Venez. 46: 174-187.
Ochoa, J. 2000. Efectos de la extracción de maderas sobre la diversidad de mamíferos pequeños en bosques de tierras bajas de la Guayana venezolana. Biotropica 32: 146-164.

Ochoa, J., M. O'Farrell \& B. Miller. 2000. Contribution of acoustic methods to the study of insectivorous bat diversity in protected areas from northern Venezuela. Acta Chiropterol. 2: 171-183.

Ochoa, J. \& M. Aguilera. 2003. Mamíferos, p. 650-672. In M. Aguilera, E. González G. \& A. Azocar. La Diversidad Biológica en Venezuela. Fundación Polar, Ministerio de Ciencia y Tecnología, Fondo Nacional de Ciencia, Tecnología e Innovación (FONACIT), Caracas, Venezuela.

Ochoa, J., M. Bevilacqua \& F. García. 2005. Evaluación ecológica rápida de las comunidades de mamíferos en cinco localidades del Delta del Orinoco, Venezuela. Interciencia 30: 466-475.

O'Farrell, M. 1999. Use of vocal signaturas for the inventory of free-flying Neotropical bats. Biotropica 31: 507-516.

O’Farrell, M. \& W. Gannon. 1999. A comparision of acoustic versus capture techniques for the inventory of bats. J. Mammal. 80: 24-30.

O’Farrell, M., B. Miller \& W. Gannon. 1999. Qualitative identification of free-flying bats using the Anabat Detector. J. Mammal. 80: 11-23.

Ojasti, J. \& E. Mondolfi. 1968. Esbozo de la fauna de mamíferos de Caracas, p. 409-461. In Universidad Central de Venezuela. Estudio de Caracas: Ecología vegetal y fauna. Imprenta Universitaria, Caracas, Venezuela.

Observatorio Mundial de los Bosques (OMB). 2002. Situación de los bosques en Venezuela: La región Guayana como caso de estudio. World Resources Institute, Global Forest Watch, ACOANA, UNEG, Provita, Caracas, Venezuela.

Oria, F. 2007. Dispersión de semillas por murciélagos en ambientes con diferentes grados de intervención en el Sector Papelón de la Sierra de Aroa, Estado Yaracuy. Tesis de licenciatura, Universidad de Carabobo, Valencia, Venezuela.

Patterson, B., V. Pacheco \& S. Solari. 1996. Distribution of bats along an elevational gradient in the Andes of South-eastern Peru. J. Zool. 240: 637-658.

Presley, S., M. Willig, J. Wunderle \& L. Saldanha. 2008. Effects of reduced-impact logging and forest 
physiognomy on bat populations of lowland Amazonian forest. J. Appl. Ecol. 45: 14-25.

Rhabek, C. 1995. The elevation gradient of species richness: a uniform pattern? Ecography 18: 200-205.

Rivas, B. \& M. Salcedo. 2005. Lista actualizada de los mamíferos del Parque Nacional El Ávila, Venezuela. Mem. Soc. Cien. Nat. La Salle 164: 29-56.

Rivas, B., A. Ferrer \& F. García. 2008. Mamíferos, p. 177196. In C. Lasso \& J. Señaris. Diversidad animal del caño Macareo, Punta Pescador y áreas adyacentes, Delta del Orinoco. La Salle, Caracas, Venezuela.

Rivas-Rojas, E. 2005. Diversity bats of dry forest and cocoa plantation. Lyona J. Ecol. Application 8: 29-39.

Rodríguez-Olarte, D., A. Amaro, J. Coronel \& D. Taphorn. 2005. Los peces del río Aroa, cuenca del Caribe, Venezuela. Mem. Soc. Cien. Nat. La Salle 164: 101-127.

Rodríguez, J. \& F. Rojas-Suárez. 2008. Libro rojo de la fauna venezolana. Provita y Shell Venezuela, S.A., Caracas, Venezuela.

Roze, J. \& H. Solano. 1963. Resumen de la familia Caecilidae (Amphibia: Gymnophiona) de Venezuela. Acta Biológica Venezuélica 3: 287-300.

Sampaio, E., E. Kalko, E. Bernard, B. Rodríguez-Herrera \& C. Handley. 2003. A biodiversity assessment of bats (Chiroptera) in a tropical lowland rainforest of central Amazonia, including methodological and conservation considerations. Stud. of Neo. Fau. and Env. 38: 17-31.

Sánchez, J., B. Rivas, H. Rojas, D. Lew, M. Salazar, A. González, M. de Lourdes \& E. Rondón. 2007. Mamíferos de Venezuela: su representatividad en colecciones nacionales. Libro de Resúmenes del VII Congreso Venezolano de Ecología. La sociedad es parte del ecosistema. Ciudad Guayana, Venezuela.
Simmons, N. \& R. Voss. 1998. The mammals of Paracou, French Guiana: a neotropical lowland rainforest fauna. Part I, Bats. Bull. Am. Mus. Nat. Hist. 237: 1-219.

Simmons, N. 2005. Order Chiroptera, p. 312-529. In D. Wilson \& D. Reeder. Mammals species of the world: a taxonomic and geographic reference. Volumen 1. The Hopkins University, Baltimore, EEUU.

Soriano, P. 1983. La comunidad de quirópteros de las selvas nubladas en los Andes de Mérida. Patrón reproductivo de los frugívoros y las estrategias ecológicas fenológicas de las plantas. Tesis de Maestría, Universidad de Los Andes, Mérida, Venezuela.

Soriano, P., A. Díaz, J. Ochoa \& M. Aguilera. 1999. Biogeographic analysis of the mammal communities in the venezuelan Andes. Interciencia 24: 17-25.

Soriano, P. 2000. Functional structure of bat communities in tropical rainforests and andean cloud forests. Ecotropicos 13: 1-20.

Soriano, P. \& J. Ochoa. 2001. The consequences of timber exploitation for bats communities in tropical America, p. 153-166. In R. Fimbel, A. Grajal \& J. Robinson. The cutting edge: conserving wildlife in logged tropical forests. Columbia University, Nueva York, EEUU.

Voss, R., D. Lunde \& N. Simmons. 2001. The mammals of Paracou, French Guiana: a neotropical lowland rainforest fauna. Part II: Nonvolant species. Bull. Am. Mus. Nat. Hist. 263: 1-236.

Willig, M., S. Presley, C. Bloch, C. Hice, S. Yanoviak, M. Díaz, L. Chauca, V. Pacheco \& S. Weaver. 2007. Phyllostomid bats of lowland Amazonia: Effects of habitat alteration on abundance. Biotropica 39: 737-746.

Wilson, D., C. Ascorra \& S. Solari. 1996. Bats as indicators of habitat disturbance, p. 613-625. In D. Wilson \& A. Sandobal. Manu, the biodiversity of southeastern Peru. Horizonte, Lima, Peru. 
APÉNDICE

Número y proporción de individuos capturados en las tres localidades inventariadas para el Parque Nacional Yurubí, Yaracuy

APPENDIX

Number and proportion of captured individuals in the three localities inventoried at the Yurubí National Park, Yaracuy

\begin{tabular}{|c|c|c|c|c|c|c|c|c|}
\hline & \multicolumn{2}{|c|}{$\begin{array}{c}\text { Mayorica } \\
(100-220 \mathrm{~m})\end{array}$} & \multicolumn{2}{|c|}{$\begin{array}{c}\text { Bernabó } \\
(400-550 \mathrm{~m})\end{array}$} & \multicolumn{2}{|c|}{$\begin{array}{c}\text { Silencio } \\
(1414-1460 \mathrm{~m})\end{array}$} & \multirow[t]{2}{*}{ Total } & \multirow[t]{2}{*}{ Acrónimo } \\
\hline & $\mathrm{N}$ ind. & $\%$ & $\mathrm{~N}$ ind. & $\%$ & $\mathrm{~N}$ ind. & $\%$ & & \\
\hline \multicolumn{9}{|l|}{ Emballonuridae } \\
\hline Saccopteryx bilineata & 1 & 0.3 & 1 & 0.3 & 0 & 0.0 & 2 & S. bil \\
\hline Saccopteryx leptura & 1 & 0.3 & 0 & 0.0 & 0 & 0.0 & 1 & S.lep \\
\hline \multicolumn{9}{|l|}{ Phyllostomidae } \\
\hline Anoura caudifera & 1 & 0.3 & 3 & 0.9 & 0 & 0.0 & 4 & A. cau \\
\hline Anoura cultrata & 0 & 0.0 & 0 & 0.0 & 2 & 1.6 & 2 & A.cul \\
\hline Anoura geoffroyi & 0 & 0.0 & 2 & 0.6 & 1 & 0.8 & 3 & A. geo \\
\hline Artibeus bogotensis & 3 & 0.8 & 5 & 1.4 & 0 & 0.0 & 8 & A. bog \\
\hline Artibeus lituratus & 29 & 7.8 & 71 & 20.4 & 19 & 14.8 & 119 & A. lit \\
\hline Artibeus planirostris & 47 & 13 & 5 & 1.4 & 20 & 15.6 & 72 & A.pla \\
\hline Carollia brevicauda & 1 & 0.3 & 8 & 2.3 & 9 & 7.0 & 18 & C. bre \\
\hline Carollia perspicillata & 111 & 30 & 105 & 30.2 & 2 & 1.6 & 218 & C.per \\
\hline Choeroniscus godmani & 0 & 0.0 & 1 & 0.3 & 0 & 0.0 & 1 & C. god \\
\hline Chrotopterus auritus & 1 & 0.3 & 0 & 0.0 & 0 & 0.0 & 1 & C. aur \\
\hline Diphylla ecaudata & 2 & 0.5 & 0 & 0.0 & 0 & 0.0 & 2 & D. eca \\
\hline Enchisthenes hartii & 0 & 0.0 & 6 & 1.7 & 3 & 2.3 & 9 & E. har \\
\hline Glossophaga soricina & 20 & 5.4 & 22 & 6.3 & 0 & 0.0 & 42 & G. sor \\
\hline Glyphonycteris sylvestris & 2 & 0.5 & 0 & 0.0 & 0 & 0.0 & 2 & G. syl \\
\hline Lampronycteris brachyotis & 3 & 0.8 & 0 & 0.0 & 0 & 0.0 & 3 & L. bra \\
\hline Lonchorhina aurita & 2 & 0.5 & 0 & 0.0 & 0 & 0.0 & 2 & L. aur \\
\hline Lophostoma brasiliense & 2 & 0.5 & 0 & 0.0 & 0 & 0.0 & 2 & L. bra \\
\hline Lophostoma silvicolum & 1 & 0.3 & 1 & 0.3 & 0 & 0.0 & 2 & L. sil \\
\hline Micronycteris megalotis & 1 & 0.3 & 0 & 0.0 & 0 & 0.0 & 1 & M.mes \\
\hline Micronycteris microtis & 1 & 0.3 & 0 & 0.0 & 1 & 0.8 & 2 & M. dic \\
\hline Micronycteris hirsuta & 4 & 1.1 & 0 & 0.0 & 0 & 0.0 & 4 & M. hir \\
\hline Micronycteris schmidtorum & 1 & 0.3 & 0 & 0.0 & 0 & 0.0 & 1 & M. sch \\
\hline Mimon crenulatum & 2 & 0.5 & 0 & 0.0 & 0 & 0.0 & 2 & M. cre \\
\hline Phylloderma stenops & 1 & 0.3 & 0 & 0.0 & 0 & 0.0 & 1 & P. sre \\
\hline Phyllostomus discolor & 8 & 2.2 & 0 & 0.0 & 0 & 0.0 & 8 & P. dis \\
\hline Phyllostomus hastatus & 2 & 0.5 & 0 & 0.0 & 0 & 0.0 & 2 & P. has \\
\hline Platyrrhinus dorsalis & 0 & 0.0 & 0 & 0.0 & 5 & 3.9 & 5 & P.dor \\
\hline Platyrrhinus helleri & 27 & 7.3 & 21 & 6.0 & 0 & 0.0 & 48 & P. hel \\
\hline Sphaeronycteris toxophyllum & 1 & 0.3 & 0 & 0.0 & 0 & 0.0 & 1 & S.tox \\
\hline Sturnira lilium & 46 & 12 & 16 & 4.6 & 2 & 1.6 & 64 & S. lil \\
\hline Sturnira oporaphilum & 9 & 2.4 & 0 & 0.0 & 22 & 17.2 & 31 & S. opor \\
\hline Tonatia saurophila & 5 & 1.3 & 0 & 0.0 & 0 & 0.0 & 5 & T. sau \\
\hline Uroderma bilobatum & 19 & 5.1 & 5 & 1.4 & 0 & 0.0 & 24 & U. bil \\
\hline Vampyressa thyone & 1 & 0.3 & 0 & 0.0 & 0 & 0.0 & 1 & V. thy \\
\hline
\end{tabular}


APÉNDICE (Continuación)

Número y proporción de individuos capturados en las tres localidades inventariadas para el Parque Nacional Yurubí, Yaracuy

APPENDIX (Continued)

Number and proportion of captured individuals in the three localities inventoried at the Yurubí National Park, Yaracuy

\begin{tabular}{|c|c|c|c|c|c|c|c|c|}
\hline & \multicolumn{2}{|c|}{$\begin{array}{c}\text { Mayorica } \\
(100-220 \mathrm{~m})\end{array}$} & \multicolumn{2}{|c|}{$\begin{array}{c}\text { Bernabó } \\
(400-550 \mathrm{~m})\end{array}$} & \multicolumn{2}{|c|}{$\begin{array}{c}\text { Silencio } \\
(1414-1460 \mathrm{~m})\end{array}$} & \multirow[t]{2}{*}{ Total } & \multirow[t]{2}{*}{ Acrónimo } \\
\hline & $\mathrm{N}$ ind. & $\%$ & $\mathrm{~N}$ ind. & $\%$ & $\mathrm{~N}$ ind. & $\%$ & & \\
\hline \multicolumn{9}{|l|}{ Mormoopidae } \\
\hline Pteronotus davyi & 0 & 0.0 & 0 & 0.0 & 20 & 15.6 & 20 & P. dav \\
\hline Pteronotus gymnonotus & 0 & 0.0 & 0 & 0.0 & 1 & 0.8 & 1 & P. gym \\
\hline Pteronotus parnellii & 8 & 2.2 & 29 & 8.3 & 11 & 8.6 & 48 & P. par \\
\hline Pteronotus personatus & 2 & 0.5 & 0 & 0.0 & 3 & 2.3 & 5 & P.per \\
\hline \multicolumn{9}{|l|}{ Vespertilionidae } \\
\hline Eptesicus furinalis & 2 & 0.5 & 12 & 3.4 & 4 & 3.1 & 18 & E. fur \\
\hline Rhogeessa io & 2 & 0.5 & 0 & 0.0 & 0 & 0.0 & 2 & R. io \\
\hline Myotis keaysi & 1 & 0.3 & 0 & 0.0 & 3 & 2.3 & 4 & M. kea \\
\hline Myotis nigricans & 1 & 0.3 & 11 & 3.2 & 0 & 0.0 & 12 & M. nig \\
\hline \multicolumn{9}{|l|}{ Molossidae } \\
\hline Cynomops greenhalli & 0 & 0.0 & 18 & 5.2 & 0 & 0.0 & 18 & C.gre \\
\hline Molossus molossus & 0 & 0.0 & 1 & 0.3 & 0 & 0.0 & 1 & M. mol \\
\hline Molossus pretiosus & 0 & 0.0 & 5 & 1.4 & 0 & 0.0 & 5 & M.pre \\
\hline \multirow[t]{2}{*}{ Total } & 371 & & 348 & & 128 & & 847 & \\
\hline & 37 spp. & & 21 spp. & & 17 spp. & & & \\
\hline
\end{tabular}

